\title{
Clinical Study Effects of Differing Dosages of Pomegranate Juice Supplementation after Eccentric Exercise
}

\author{
Daniel R. Machin, Kevin M. Christmas, Ting-Heng Chou, Sarah C. Hill, \\ Douglas W. Van Pelt, Justin R. Trombold, and Edward F. Coyle
}

The Human Performance Laboratory, Department of Kinesiology and Health Education, The University of Texas at Austin, One University Station, Bellmont Hall, D3700, Austin, TX 78712, USA

Correspondence should be addressed to Edward F. Coyle; coyle@austin.utexas.edu

Received 24 July 2014; Accepted 16 September 2014; Published 29 September 2014

Academic Editor: Charles Dumke

Copyright (C) 2014 Daniel R. Machin et al. This is an open access article distributed under the Creative Commons Attribution License, which permits unrestricted use, distribution, and reproduction in any medium, provided the original work is properly cited.

\begin{abstract}
Dietary supplementation with pomegranate juice improves isometric strength recovery after unaccustomed eccentric exercise. The purpose of this study was to determine if there is a dose response effect of pomegranate juice supplementation after eccentric exercise isometric strength recovery. Forty-five nonresistance trained, recreationally active men were assigned once-daily pomegranate juice, twice-daily pomegranate juice, or placebo supplementation. On day four of supplementation, 20 min of downhill running and 40 maximal eccentric elbow flexion repetitions were performed. Isometric knee extensor and elbow flexor strength, muscular soreness, and serum myoglobin concentrations were measured prior to exercise and 2, 24, 48, 72, and $96 \mathrm{~h}$ after exercise. Throughout the postexercise time period, while isometric knee extensor and elbow flexor strength were similar between once-daily and twice-daily pomegranate juice supplementation groups, isometric strength was significantly higher in pomegranate juice groups than placebo. Knee extensor soreness, elbow flexor soreness, and myoglobin increased in response to exercise but were similar between groups. It is apparent that pomegranate juice supplementation improves strength recovery in leg and arm muscles following eccentric exercise; however, no dose response effect was present. We conclude that once-daily pomegranate juice supplementation is not different from twice-daily supplementation in regards to strength recovery after eccentric exercise.
\end{abstract}

\section{Introduction}

The performance of an acute bout of unaccustomed eccentric exercise results in immediate muscle damage and reductions in maximal strength of the exercising muscles [1]. During the 24-48-hour time period after eccentric exercise, muscular soreness peaks, after which soreness begins to return to baseline values [2]. However, maximal strength remains lower than baseline for several days [3]. This condition, delayed onset muscle soreness (DOMS), occurs in individuals who are unaccustomed to performing a high volume or intensity of eccentric contractions, but it can also occur in resistance trained individuals [4].

A goal of numerous studies has been to identify dietary supplements that accelerate recovery from a bout of intense eccentric exercise [4-20]. Antioxidant and nonsteroidal antiinflammatory drugs (NSAIDs) have a negligible effect on strength recovery [5-13]. On the contrary, NSAID [10] and vitamin $C[14,15]$ supplementation have been shown to alleviate muscle soreness. Dietary supplementation with polyphenols, a class of antioxidant and anti-inflammatory phytonutrients [21], has been demonstrated to improve muscular strength recovery after eccentric exercise in humans $[4,16-20]$.

The pomegranate fruit contains an abundance of polyphenols [21]. Pomegranate juice supplementation elicits an ergogenic effect in both untrained and, to a lesser extent, resistance trained individuals after eccentric exercise $[4$, 19]. In these studies, participants consumed twice-daily pomegranate supplementation, corresponding to a daily polyphenol supplementation dose of 1,300 $\mathrm{mg}$ gallic acid equivalents (GAE) and displayed an accelerated recovery of isometric strength with the polyphenol supplementation compared to placebo. 
The purpose of this study was to determine if there is a dose response effect of pomegranate polyphenol supplementation on muscular strength recovery following a bout of combined upper and lower body eccentric exercise in nonresistance trained, recreationally active participants. The doses of pomegranate polyphenol supplementation provided in the present study were $1,300 \mathrm{mg}$ or $650 \mathrm{mg} \mathrm{GAE} / \mathrm{d}$ and correspond to the same or half the dose used in previous pomegranate supplementation studies, respectively $[4,19]$. The present study employed nonresistance trained, recreationally active individuals because they have previously been shown to display a robust ergogenic effect to $1,300 \mathrm{mg} \mathrm{GAE} / \mathrm{d}$.

\section{Methods}

2.1. Participants. Forty-five healthy, nonsmoking, nonresistance trained, recreationally active young men (22.3 \pm 4.1 years, $73.8 \pm 11.5 \mathrm{~kg}, 174.9 \pm 6.2 \mathrm{~cm}$ ) were recruited to participate in this study. Participants provided written informed consent to participate in the experimental protocol approved by the university Institutional Review Board. Prior to entrance into the study, participants completed a health history questionnaire and indicated no history of orthopedic injury and had not participated in any resistance training exercise, distance running, or activity that resulted in muscle soreness in the previous six months. Additional exclusion criteria were hypertension, kidney dysfunction, active weight loss $>5 \mathrm{~kg}$ in the prior three months, lipid-lowering drugs, or selective serotonin reuptake inhibitors.

2.2. Study Design. This was a double-blind, randomized, placebo-controlled study with one testing period lasting eight days (Figure 1). One week prior to and throughout the experimental protocol participants discontinued all antiinflammatory, antioxidant, and multivitamin supplements. Additionally, participants completed a 3-day familiarization period to practice isometric strength tests to ensure that each participant was comfortable and able to generate maximal isometric strength. After familiarization, participants were randomized into one of three supplementation groups: (1) once-daily (650 mg GAE/d; 1x) serving of pomegranate juice concentrate (PJC), (2) twice-daily (1,300 mg GAE/d; 2x) PJC, or (3) placebo (0 mg GAE/d; PLA). Eccentric exercise was performed on day four of the 8-day supplementation period with baseline collection of blood, soreness, and strength data made prior to exercise and repeated 2, 24, 48, 72, and $96 \mathrm{~h}$ after exercise. On all subsequent visits participants reported to the laboratory in a similar postprandial state and were instructed to avoid caffeine consumption.

2.3. Dietary Supplementation. Supplements were consumed twice-daily at 12-hour intervals. In the $1 x$ treatment participants consumed PJC in the morning and PLA in the evening, in the $2 \mathrm{x}$ treatment they consumed PJC in both morning and evening, and in the PLA group they consumed PLA in both morning and evening. Supplement drinks were provided as a concentrate stored in $500 \mathrm{~mL}$ bottles using the Wonderful variety pomegranates grown in California
(POM Wonderful Inc., LLP; Los Angeles, CA). Participants diluted $30 \mathrm{~mL}$ servings of POM or PLA with water and consumed the drinks in one sitting. Each PJC serving contained $650 \mathrm{mg} \mathrm{GAE}$ /serving of pomegranate polyphenols consisting of $95.5 \%$ ellagitannins, 3.5\% ellagic acid, and $1 \%$ anthocyanins. Both PJC and PLA contained 24 grams of carbohydrate/serving with additional coloring and flavoring used to blind the treatments. Participants were reminded verbally and through e-mail communication to consume the experimental supplements at the required times.

2.4. Eccentric Exercise. Eccentric exercise included $20 \mathrm{~min}$ of downhill running followed by 40 repetitions of bilateral isotonic eccentric contractions of the elbow flexors. Ten sets of downhill running ( $2 \mathrm{~min}$ per set) were performed outdoors on the concrete pedestrian ramps of Darrell $\mathrm{K}$. Royal-Texas Memorial Stadium. The total downhill running distance was $4 \mathrm{~km}$ at a $-10 \%$ grade. A member of the research team accompanied each subject to record the time taken to perform each set and help maintain a predetermined velocity of $\sim 200 \mathrm{~m} / \mathrm{min}$. At the end of each set, lasting approximately $2 \mathrm{~min}(400 \mathrm{~m})$, an elevator was used to take the participant back to the top floor to perform the subsequent set, until 10 sets were completed.

After the final set of downhill running, 40 repetitions of bilateral isotonic eccentric contractions of the elbow flexors were performed on a preacher curl bench. A member of the research team placed an EZ curl barbell in the participant's hands with the elbow at $130^{\circ}$ of elbow flexion and the participant slowly lowered the barbell until reaching $0^{\circ}$ of elbow flexion. Each repetition was 5 seconds in length and repeated 40 times with 20 seconds rest between repetitions. The weight used corresponded to each participant's concentric 1-repetition maximum (1-RM). 1-RM measurement was performed on the same day as eccentric exercise by having participants perform one bilateral concentric elbow flexion repetition with a weight corresponding to approximately $80 \%$ of their maximal isometric elbow flexor strength. If successful, the weight was increased until a repetition could not be performed.

2.5. Isometric Strength. Isometric knee extensor strength measurement was performed bilaterally using a modified knee extension apparatus with participants secured by waist and shoulder straps. Strength was recorded using a load cell (LC101-500, Omega Engineering, Stamford, CT) secured to the base of the apparatus with a galvanized steel cable secured to the lower legs of the participant. Each participant performed three trials at $65^{\circ}$ knee flexion with 2 min rest between trials.

Isometric elbow flexor strength measurement was performed bilaterally while seated on a modified preacher bench. Strength was recorded using a load cell (LC101-500, Omega Engineering, Stamford, CT) secured to the ground using a galvanized steel cable and a "curl" bar that was held in the participant's hands. Each participant performed three trials at $45^{\circ}$ elbow flexion with 2 min rest between trials. Isometric strength was obtained from the peak value of three trials 


\begin{tabular}{|c|c|c|c|c|c|c|c|c|c|}
\hline \multirow{2}{*}{$\begin{array}{c}\text { Three-day } \\
\text { familiarization } \\
\text { period }\end{array}$} & \multicolumn{9}{|c|}{ Twice-daily dietary supplementation ( $1 \mathrm{x}, 2 \mathrm{x}$, or PLA) } \\
\hline & Day 1 & Day 2 & Day 3 & & & Day 5 & Day 6 & Day 7 & Day 8 \\
\hline & & & & & $2 \mathrm{~h}$ & $24 \mathrm{~h}$ & $48 \mathrm{~h}$ & $72 \mathrm{~h}$ & $96 \mathrm{~h}$ \\
\hline
\end{tabular}

FIGURE 1: Experimental protocol. Eccentric exercise was performed on day four of the 8-day supplementation period with baseline collection of blood, soreness, and strength made prior to exercise and repeated 2, 24, 48, 72, and $96 \mathrm{~h}$ after exercise.

at each time point and reported as a percentage of baseline values.

2.6. Muscle Soreness. Soreness of the knee extensor and elbow flexor muscles was determined by having participants rate the degree of soreness using a visual analog scale of 0 to 10 , with 0 described as "no soreness" and 10 described as "unbearable soreness." Soreness obtained while performing one repetition of unloaded elbow flexion and an unloaded squat to a predetermined depth.

2.7. Blood Collection and Analysis. Blood was collected from the antecubital vein into vacuum-sealed tubes (Vacutainer, Franklin Lakes, NJ) and allowed to clot. After which, serum tubes were centrifuged at $1,500 \times \mathrm{g}$ for $10 \mathrm{~min}$ at $4^{\circ} \mathrm{C}$, allocated into several microcentrifuge collection tubes, and stored at $-80^{\circ} \mathrm{C}$. Serum was analyzed for myoglobin using a commercially available assay kit (Oxis International, Foster City, CA). Samples were centrifuged at $17,000 \times \mathrm{g}$ for $5 \mathrm{~min}$ at $4^{\circ} \mathrm{C}$ before analysis.

2.8. Myoglobin. Myoglobin concentration was measured in duplicate using the enzyme-linked immunosorbent method. Samples were allocated into antibody-coated wells that bind myoglobin, after which, a secondary antibody is added, which binds to the myoglobin, which contains an enzyme subunit generating a color change when the final photosensitive chemicals are added. The assay is measured using a spectrophotometer plate reader at $450 \mathrm{~nm}$.

2.9. Statistical Analyses. Data were analyzed with a twoway ANOVA for treatment, time, and treatment $\times$ time interactions. Significance was assessed at alpha level $<0.05$. All analyses were performed using SPSS software (Chicago, IL). Values were reported as Mean \pm SD.

\section{Results}

There was no difference in downhill running velocity between 1x, 2x, and PLA (204 \pm 4, $205 \pm 5,207 \pm 4 \mathrm{~m} / \mathrm{min}$, resp.; $P>0.05)$. When averaged throughout the postexercise time period, there were significant differences in isometric knee extensor strength between $1 \mathrm{x}, 2 \mathrm{x}$, and PLA (treatment effect, $93.9 \pm 6.1,91.6 \pm 5.6,87.1 \pm 7.9 \%$ of baseline, resp.; $P<$ 0.05). Both $1 \mathrm{x}$ and $2 \mathrm{x}$ PJC treatments resulted in significantly

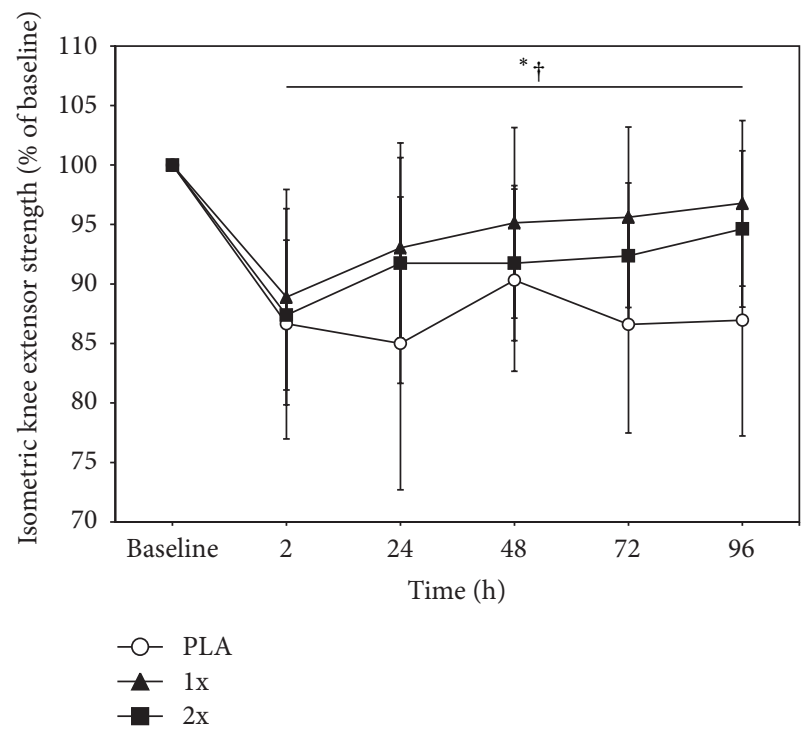

FIGURE 2: Isometric knee extensor strength reported as a percent of baseline values $(n=45)$. Treatments were oncedaily (650 mg GAE/d) (1x; filled triangles) or twice-daily (1,300 mg GAE/d) (2x; filled squares) pomegranate juice concentrate or placebo (PLA; open circles). ${ }^{*} 1 \mathrm{x}$ significantly higher than PLA, main treatment effect $(P<0.05) .{ }^{\dagger} 2 \mathrm{x}$ significantly higher than PLA, main treatment effect $(P<0.05)$. Values reported as Mean \pm SD.

higher isometric knee extensor strength than PLA $(P<0.05)$ (Figure 2). There was a significant time effect for isometric knee extensor strength $(P<0.05)$, in which strength was lower than baseline at each time point after eccentric exercise. There was no significant interaction effect for isometric knee extensor strength $(P>0.05)$ nor were there any significant interaction effects for any measured variable in the present study. Soreness of knee extensor muscles was not different between $1 \mathrm{x}, 2 \mathrm{x}$, and PLA in the postexercise time period $(2.3 \pm 1.3,2.3 \pm 1.1,2.3 \pm 1.4$, resp.; $P>0.05)$. There was a significant time effect for knee extensor soreness $(P<0.05)$, peaking 24-48 h after eccentric exercise (Figure 3).

All participants completed 40 repetitions of eccentric elbow flexion. There was no difference in elbow flexor concentric 1-RM strength between $1 x, 2 x$, and PLA ( $32 \pm 8$, $31 \pm 8,31 \pm 7 \mathrm{~kg}$, resp.; $P>0.05$ ). Throughout the postexercise time period, there were significant differences in isometric elbow flexor strength between $1 x, 2 x$, and PLA (treatment 


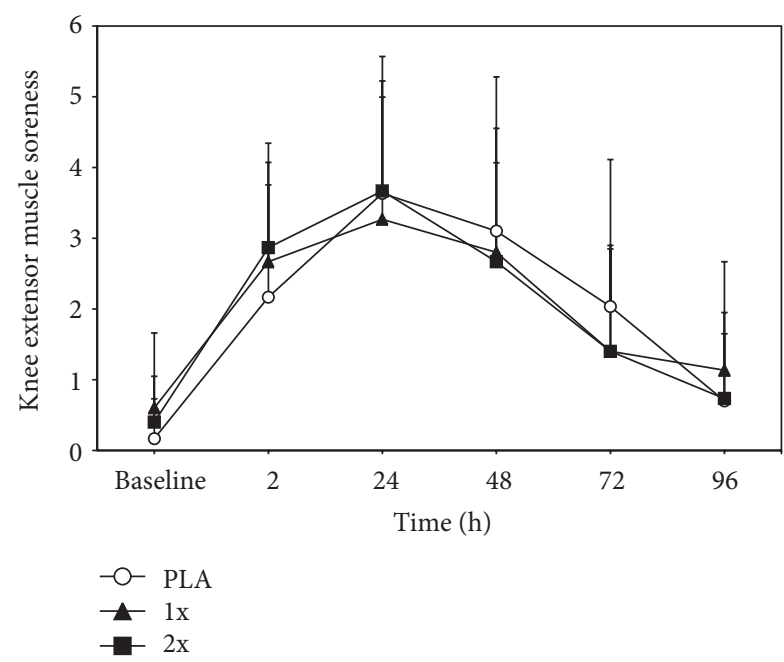

FIGURE 3: Muscle soreness of knee extensor muscles reported on a scale of 0 to $10(n=45)$. Treatments were oncedaily (650 mg GAE/d) (1x; filled triangles) or twice-daily (1,300 mg GAE/d) (2x; filled squares) pomegranate juice concentrate or placebo (PLA; open circles). Values reported as Mean \pm SD.

effect, $83.6 \pm 10.4,85.6 \pm 7.2,78.4 \pm 6.7 \%$ of baseline, resp.; $P<$ $0.05)$. Both $1 \mathrm{x}$ and $2 \mathrm{x}$ PJC treatments resulted in significantly higher isometric elbow flexor strength than PLA $(P<0.05)$ (Figure 4). There was a significant time effect for isometric elbow flexor strength $(P<0.05)$, in which strength was lower than baseline at each time point after eccentric exercise. Soreness of elbow flexor muscles was not different between $1 \mathrm{x}$, $2 \mathrm{x}$, and PLA in the postexercise time period (3.0 $\pm 1.5,2.9 \pm 1.1$, $2.9 \pm 1.2$, resp.; $P>0.05$ ). There was a significant time effect for elbow flexor soreness $(P<0.05)$, peaking $24-48 \mathrm{~h}$ after eccentric exercise (Figure 5).

There was no difference in myoglobin between $1 \mathrm{x}, 2 \mathrm{x}$, and PLA in the postexercise time period $(79 \pm 56,53 \pm 27$, $56 \pm 22 \mathrm{ng} / \mathrm{mL}$, resp.; $P>0.05$ ) (Figure 6). There was a significant time effect for myoglobin $(P<0.05)$, peaking $2 \mathrm{~h}$ after eccentric exercise (Figure 6). Myoglobin data are reported after removal of outliers, which reduced sample size to $n=39$. Outliers were defined as individuals that exhibited values four times the group mean at any time point.

\section{Discussion}

The primary finding of this study was that in nonresistance trained, recreationally active men, both $1 \mathrm{x}$ and $2 \mathrm{x}$ PJC supplementation resulted in significantly higher knee extensor and elbow flexor isometric strength throughout the postexercise time period, as compared with PLA. To the best of our knowledge, this is the first study to show an ergogenic effect of pomegranate polyphenol supplementation at a dosage of $650 \mathrm{mg} \mathrm{GAE} / \mathrm{d}$. Thus, these data indicate that isometric strength recovery after eccentric exercise was not different when taking 1,300 mg GAE/d (2x) or $650 \mathrm{mg} \mathrm{GAE} / \mathrm{d}(1 \mathrm{x})$, but that with both doses isometric strength was significantly greater than PLA.

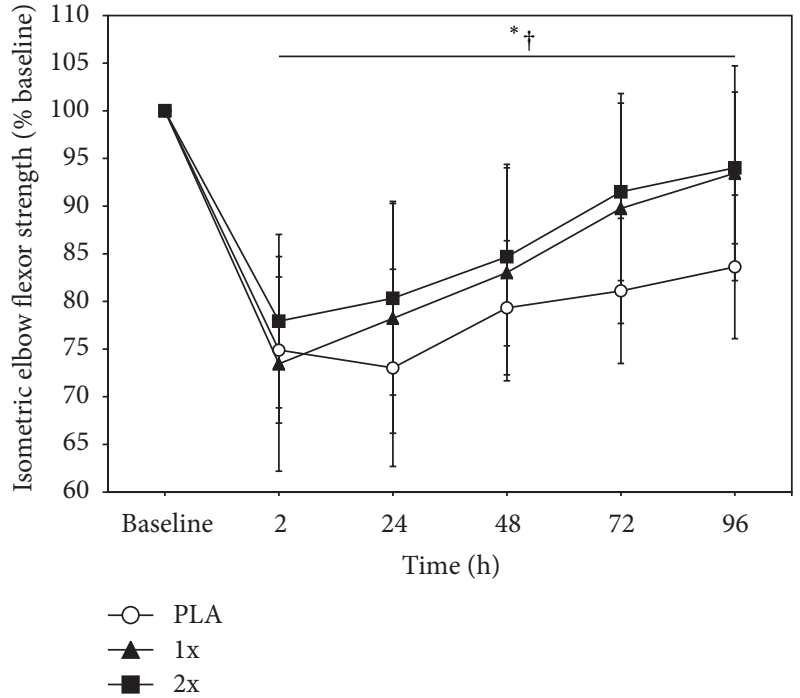

FIgURE 4: Isometric elbow flexor strength reported as a percent of baseline values $(n=45)$. Treatments were oncedaily (650 mg GAE/d) (1x; filled triangles) or twice-daily (1,300 mg GAE/d) (2x; filled squares) pomegranate juice concentrate or placebo (PLA; open circles). ${ }^{*} 1 \mathrm{x}$ significantly higher than PLA, main treatment effect $(P<0.05) .{ }^{\dagger} 2 \mathrm{x}$ significantly higher than PLA, main treatment effect $(P<0.05)$. Values reported as Mean \pm SD.

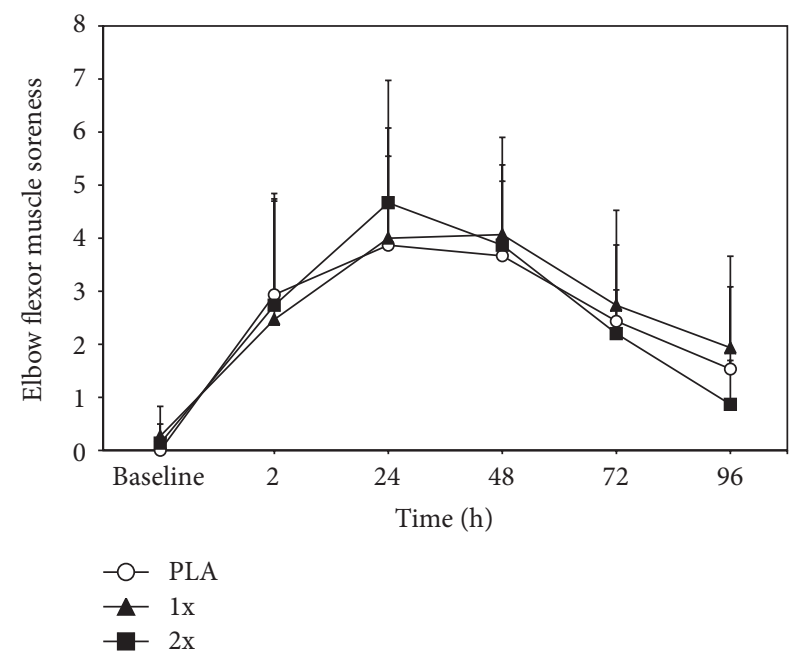

Figure 5: Muscle soreness of elbow flexor muscles reported on a scale of 0 to $10(n=45)$. Treatments were oncedaily (650 mg GAE/d) (1x; filled triangles) or twice-daily (1,300 mg GAE/d) (2x; filled squares) pomegranate juice concentrate or placebo (PLA; open circles). Values reported as Mean \pm SD.

Several studies have investigated the effects of polyphenol supplementation on isometric strength and muscle soreness following a bout of high intensity eccentric exercise $[4,19]$, resistance exercise [16], and long distance running [18, 20]. In these studies, participants had been provided twice-daily polyphenol supplementation ( 1,200-1,300 mg GAE/d) and were shown to elicit an ergogenic effect. However, it was not 


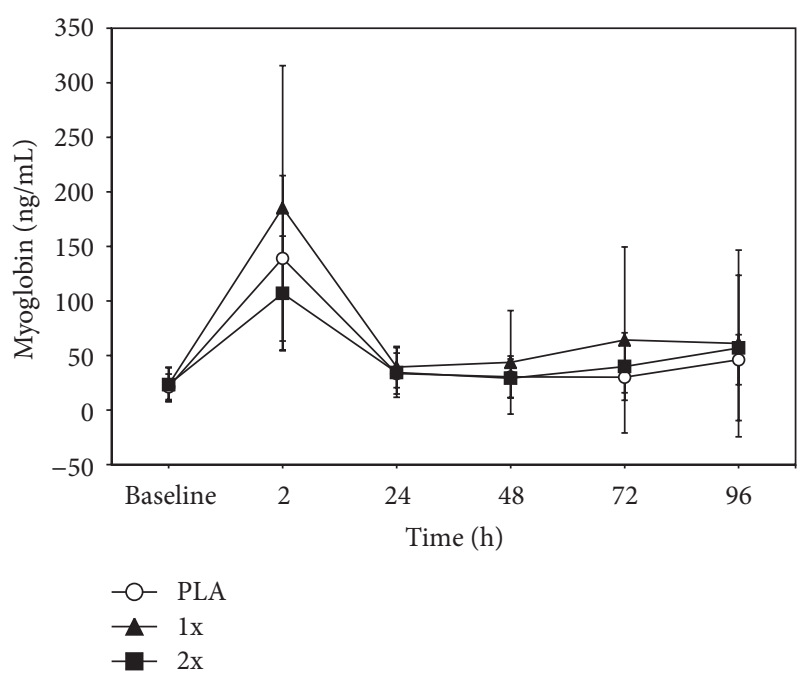

FIGURE 6: Serum myoglobin concentration $(\mathrm{ng} / \mathrm{mL} ; n=39)$. Treatments were once-daily (650 mg GAE/d) (1x; filled triangles) or twice-daily (1,300 mg GAE/d) (2x; filled squares) pomegranate juice concentrate or placebo (PLA; open circles). Values reported as Mean \pm SD.

known whether a lower daily dosage of polyphenols would be able to elicit an ergogenic effect. Therefore, the primary purpose of this study was to investigate a dose response effect of polyphenol supplementation by comparing a lower daily dosage of pomegranate polyphenol supplementation ( $650 \mathrm{mg} \mathrm{GAE} / \mathrm{d}$ ) with the same dosage used in previous studies $(1,300 \mathrm{mg} \mathrm{GAE} / \mathrm{d})$ [4, 19]. While we were unable to show a dose response effect, it appeared that a lower polyphenol dose of $650 \mathrm{mg} \mathrm{GAE} / \mathrm{d}$ was sufficient to improve strength recovery after eccentric exercise. Moreover, there were no statistical differences in isometric upper or lower body strength between the PJC supplementation groups ( $650 \mathrm{mg} \mathrm{GAE} / \mathrm{d}$ versus 1,300 mg GAE/d). Thus, we were able to demonstrate a similar ergogenic effect using a daily polyphenol dosage that was approximately half the dosage used in previous studies.

In the hours after a bout of unaccustomed eccentric exercise, muscular strength remains decreased [22, 23]. Indeed, in the present study, two hours after eccentric exercise there were significant reductions in isometric strength of elbow flexor and knee extensor muscles (25\% and 12\%, resp.), as well as increased serum myoglobin concentration, a blood marker of muscle damage. Although neither PJC treatment attenuated the rise in myoglobin in the days after exercise, it should be noted that while myoglobin is a classic blood marker of muscle damage, it correlates poorly with muscle function and cannot be used as a precise marker to quantify muscle damage [24]. On the contrary, isometric strength decrement has been shown to be an accurate noninvasive marker of muscle damage, as strength decrement is proportionate to the amount of muscle damage [25]. At no point during the 96-hour postexercise time period did isometric strength recover to baseline values. This is not surprising, as it is well established that maximal strength can remain lower than baseline for weeks after a bout of unaccustomed eccentric exercise [3]. Although isometric knee extensor or elbow flexor strength of either PJC supplementation group never returned to baseline, strength was significantly higher in both $1 \mathrm{x}$ and $2 \mathrm{x}$ PJC groups than PLA during the postexercise time period. These results indicate that muscle damage, while similar in the immediate hours after exercise, may have been recovered to a greater extent in the PJC supplementation groups in the days following eccentric exercise.

In addition to decreased muscular strength, there are increased oxidative stress and inflammation at the site of muscle damage immediately following a bout of unaccustomed eccentric exercise [26]. Pomegranate polyphenols contain an abundance of antioxidant and anti-inflammatory properties [21]; thus, a potential mechanism for the improved strength recovery observed in PJC supplementation groups may be due to attenuated oxidative stress or inflammation at some point during the postexercise time period. However, it must be stated that this may not be the case, as studies that have investigated the effects of antioxidant or antiinflammatory supplementation on strength recovery after eccentric exercise have demonstrated equivocal findings [515]. Indeed, some studies have shown a beneficial effect of antioxidant supplementation at lower dosages [11, 12], while, at higher dosages, others have shown no effect [7] or detrimental effects [9]. Thus, it is unclear if attenuating postexercise oxidative stress or inflammation is a viable mechanism for the improvements in isometric strength observed in the present study.

Strength recovery after a bout of strenuous exercise that results in reduced strength is of paramount importance to athletes, particularly those whose performance relies on optimal strength. The present findings indicate that recovery of muscular strength after a bout of upper and lower body eccentric exercise was greater in those who consumed PJC supplementation. Although participants in the present study were nonresistance trained, recreationally active men, pomegranate supplementation has also been shown to be effective in resistance trained athletes [4]. It is plausible that improved recovery could allow for higher performance in subsequent exercise bouts, leading to an accelerated accrual of training adaptations over time. However, it is unknown if pomegranate supplementation during periods of chronic training would have ergogenic effects on exercise training adaptations.

In conclusion, $1 \mathrm{x}$ (650 mg GAE/d) and 2x (1,300 mg GAE/d) PJC supplementation resulted in significantly higher isometric knee extensor and elbow flexor strength throughout the 96-hour time period after eccentric exercise compared to PLA. Furthermore, there was not a significant difference between the $1 \mathrm{x}$ and $2 \mathrm{x}$ doses of the PJC supplements. Therefore, to receive the benefits of pomegranate juice supplementation on strength recovery in both the arms and legs following bouts of eccentric exercise that will result in DOMS, only half the dosage (1x or $650 \mathrm{mg} \mathrm{GAE} / \mathrm{d}$ ) used in previous studies $[4,16,18-20]$ (2x or 1,300 $\mathrm{mg} \mathrm{GAE} / \mathrm{d}$ ) may be required. 


\section{Conflict of Interests}

The authors declare that there is no conflict of interests regarding the publication of this paper.

\section{Acknowledgments}

Daniel R. Machin, Kevin M. Christmas, Ting-Heng Chou, Sarah C. Hill, Douglas W. Van Pelt, Justin R. Trombold, and Edward F. Coyle participated in the conception and design of research. Daniel R. Machin, Kevin M. Christmas, Ting-Heng Chou, Sarah C. Hill, and Douglas W. Van Pelt participated in subject recruitment and data collection. Daniel R. Machin, Kevin M. Christmas, and Edward F. Coyle analyzed data. Daniel R. Machin, Kevin M. Christmas, Justin R. Trombold, and Edward F. Coyle interpreted results of the experiment. Daniel R. Machin and Kevin M. Christmas drafted the paper. Daniel R. Machin, Kevin M. Christmas, Ting-Heng Chou, Sarah C. Hill, Douglas W. Van Pelt, Justin R. Trombold, and Edward F. Coyle edited and approved the paper for submission. The present study was funded by a grant obtained from POM Wonderful Inc., LLP. POM Wonderful Inc., LLP did not have final approval for the content of this paper.

\section{References}

[1] M. J. Gibala, J. D. MacDougall, M. A. Tarnopolsky, W. T. Stauber, and A. Elorriaga, "Changes in human skeletal muscle ultrastructure and force production after acute resistance exercise," Journal of Applied Physiology, vol. 78, no. 2, pp. 702-708, 1995.

[2] D. J. Newham, K. R. Mills, B. M. Quigley, and R. H. T. Edwards, "Pain and fatigue after concentric and eccentric muscle contractions," Clinical Science, vol. 64, no. 1, pp. 55-62, 1983.

[3] P. M. Clarkson and M. J. Hubal, "Exercise-induced muscle damage in humans," The American Journal of Physical Medicine and Rehabilitation, vol. 81, no. 11, pp. S52-S69, 2002.

[4] J. R. Trombold, A. S. Reinfeld, J. R. Casler, and E. F. Coyle, "The effect of pomegranate juice supplementation on strength and soreness after eccentric exercise," Journal of Strength and Conditioning Research, vol. 25, no. 7, pp. 1782-1788, 2011.

[5] R. A. Fielding, T. J. Manfredi, W. Ding, M. A. Fiatarone, W. J. Evans, and J. G. Cannon, "Acute phase response in exercise III. Neutrophil and IL-1 $\beta$ accumulation in skeletal muscle," American Journal of Physiology-Regulatory Integrative and Comparative Physiology, vol. 265, no. 1, pp. R166-R172, 1993.

[6] A. Childs, C. Jacobs, T. Kaminski, B. Halliwell, and C. Leeuwenburgh, "Supplementation with vitamin $\mathrm{C}$ and $\mathrm{N}$-acetyl-cysteine increases oxidative stress in humans after an acute muscle injury induced by eccentric exercise," Free Radical Biology and Medicine, vol. 31, no. 6, pp. 745-753, 2001.

[7] L. J. Beaton, D. A. Allan, M. A. Tarnopolsky, P. M. Tiidus, and S. M. Phillips, "Contraction-induced muscle damage is unaffected by vitamin E supplementation," Medicine and Science in Sports and Exercise, vol. 34, no. 5, pp. 798-805, 2002.

[8] A. H. Goldfarb, R. J. Bloomer, and M. J. Mckenzie, “Combined antioxidant treatment effects on blood oxidative stress after eccentric exercise," Medicine and Science in Sports and Exercise, vol. 37, no. 2, pp. 234-239, 2005.

[9] G. L. Close, T. Ashton, T. Cable et al., "Ascorbic acid supplementation does not attenuate post-exercise muscle soreness following muscle-damaging exercise but may delay the recovery process," British Journal of Nutrition, vol. 95, no. 5, pp. 976-981, 2006.

[10] A. E. Donnelly, K. McCormick, R. J. Maughan, P. H. Whiting, and P. M. Clarkson, "Effects of a non-steroidal antiinflammatory drug on delayed onset muscle soreness and indices of damage," British Journal of Sports Medicine, vol. 22, no. 1, pp. 35-38, 1988.

[11] P. Jakeman and S. Maxwell, "Effect of antioxidant vitamin supplementation on muscle function after eccentric exercise," European Journal of Applied Physiology and Occupational Physiology, vol. 67, no. 5, pp. 426-430, 1993.

[12] S. R. J. Maxwell, P. Jakeman, H. Thomason, C. Leguen, and G. H. G. Thorpe, "Changes in plasma antioxidant status during eccentric exercise and the effect of vitamin supplementation," Free Radical Research Communications, vol. 19, no. 3, pp. 191202, 1993.

[13] D. A. J. Connolly, C. Lauzon, J. Agnew, M. Dunn, and B. Reed, "The effects of vitamin C supplementation on symptoms of delayed onset muscle soreness," Journal of Sports Medicine and Physical Fitness, vol. 46, no. 3, pp. 462-467, 2006.

[14] M. Kaminski and R. Boal, "An effect of ascorbic acid on delayed onset muscle soreness," Pain, vol. 50, no. 3, pp. 317-321, 1992.

[15] S. C. Bryer and A. H. Goldfarb, "Effect of high dose vitamin C supplementation on muscle soreness, damage, function, and oxidative stress to eccentric exercise," International Journal of Sport Nutrition and Exercise Metabolism, vol. 16, no. 3, pp. 270280, 2006.

[16] J. L. Bowtell, D. P. Sumners, A. Dyer, P. Fox, and K. N. Mileva, "Montmorency cherry juice reduces muscle damage caused by intensive strength exercise," Medicine and Science in Sports and Exercise, vol. 43, no. 8, pp. 1544-1551, 2011.

[17] D. A. J. Connolly, M. P. McHugh, and O. I. Padilla-Zakour, "Efficacy of a tart cherry juice blend in preventing the symptoms of muscle damage," British Journal of Sports Medicine, vol. 40, no. 8, pp. 679-683, 2006.

[18] G. Howatson, M. P. McHugh, J. A. Hill et al., "Influence of tart cherry juice on indices of recovery following marathon running," Scandinavian Journal of Medicine and Science in Sports, vol. 20, no. 6, pp. 843-852, 2010.

[19] J. R. Trombold, J. N. Barnes, L. Critchley, and E. F. Coyle, "Ellagitannin consumption improves strength recovery 2-3 d after eccentric exercise," Medicine and Science in Sports and Exercise, vol. 42, no. 3, pp. 493-498, 2010.

[20] K. S. Kuehl, E. T. Perrier, D. L. Elliot, and J. C. Chesnutt, "Efficacy of tart cherry juice in reducing muscle pain during running: a randomized controlled trial," Journal of the International Society of Sports Nutrition, vol. 7, article 17, 2010.

[21] C. Manach, G. Williamson, C. Morand, A. Scalbert, and C. Remesy, "Bioavailability and bioefficacy of polyphenols in humans. I. Review of 97 bioavailability studies," The American Journal of Clinical Nutrition, vol. 81, pp. 230S-242S, 2005.

[22] M. I. Kingsley, L. P. Kilduff, J. Mceneny, R. E. Dietzig, and D. Benton, "Phosphatidylserine supplementation and recovery following downhill running," Medicine and Science in Sports and Exercise, vol. 38, no. 9, pp. 1617-1625, 2006.

[23] J. M. Peake, K. Suzuki, G. Wilson et al., "Exercise-induced muscle damage, plasma cytokines, and markers of neutrophil activation," Medicine and Science in Sports and Exercise, vol. 37, no. 5, pp. 737-745, 2005.

[24] P. M. Clarkson, W. C. Byrnes, K. M. McCormick, L. P. Turcotte, and J. S. White, "Muscle soreness and serum creatine kinase 
activity following isometric, eccentric, and concentric exercise," International Journal of Sports Medicine, vol. 7, no. 3, pp. 152-155, 1986.

[25] G. L. Warren, D. A. Lowe, and R. B. Armstrong, "Measurement tools used in the study of eccentric contraction-induced injury," Sports Medicine, vol. 27, no. 1, pp. 43-59, 1999.

[26] F. X. Pizza, T. J. Koh, S. J. McGregor, and S. V. Brooks, "Muscle inflammatory cells after passive stretches, isometric contractions, and lengthening contractions," Journal of Applied Physiology, vol. 92, no. 5, pp. 1873-1878, 2002. 

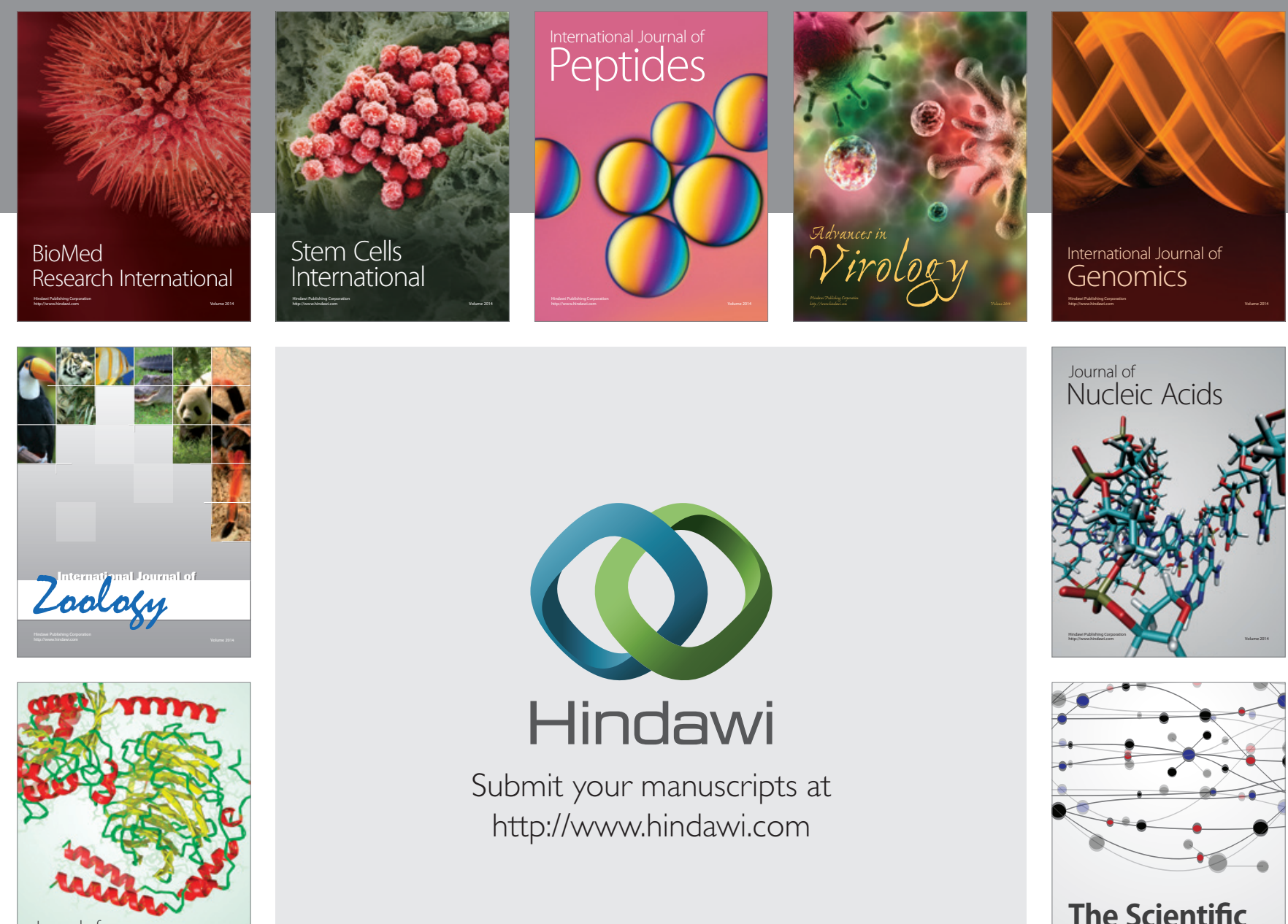

Submit your manuscripts at

http://www.hindawi.com

Journal of
Signal Transduction
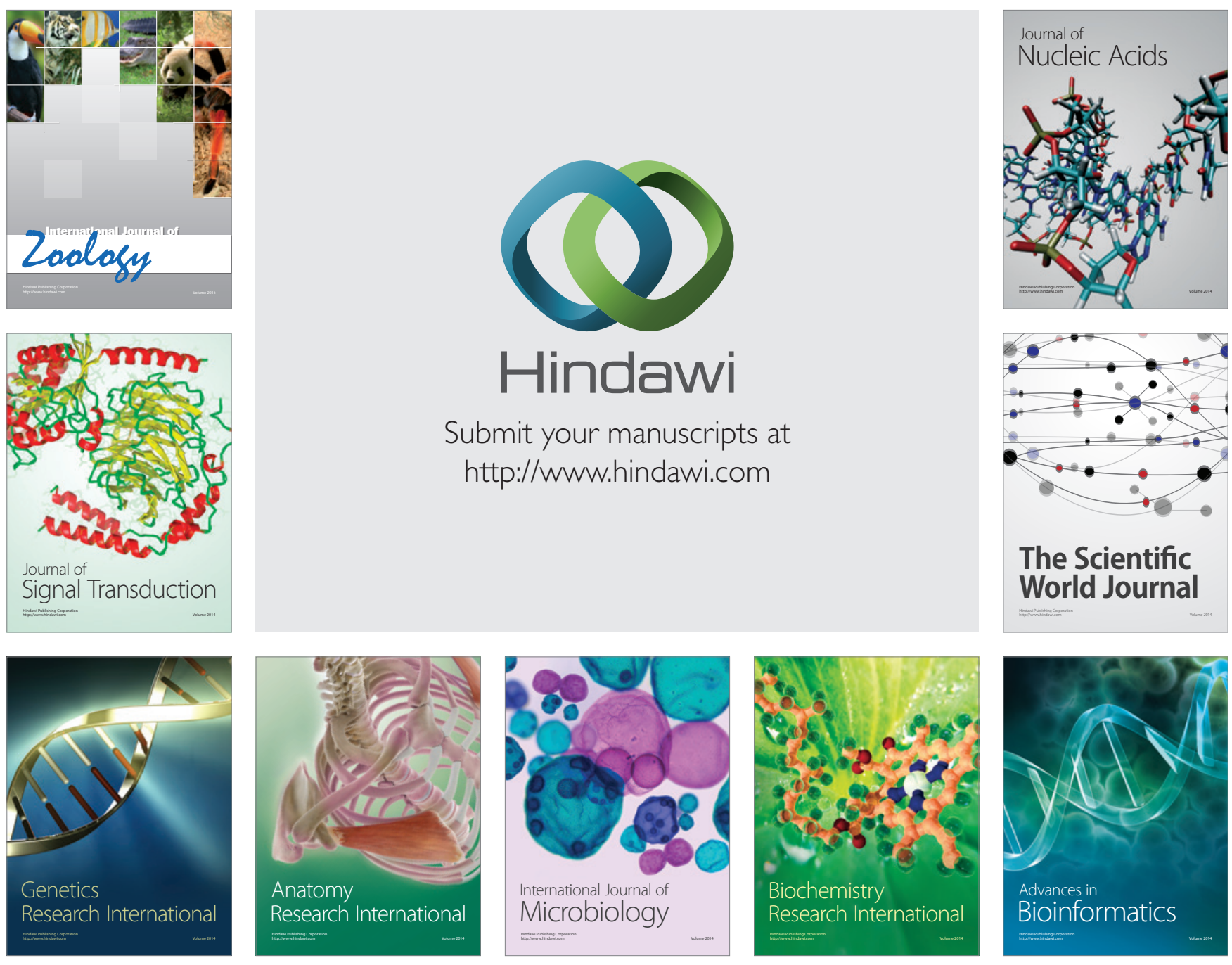

The Scientific World Journal
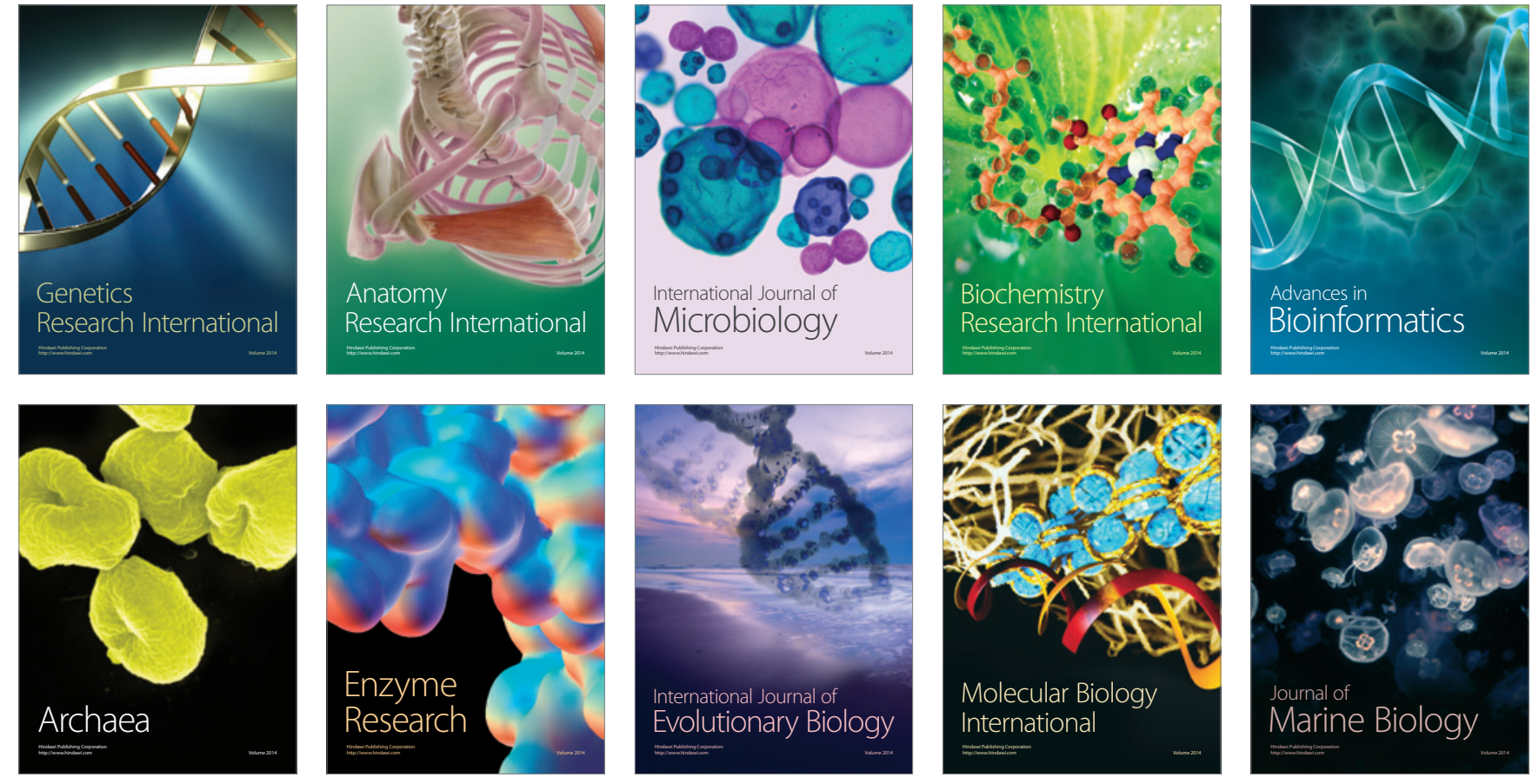